\title{
Análisis de los elementos principales en el diseño de placas de bloqueo en una fractura de 2-partes del cuello quirúrgico del húmero utilizando MEF y análisis estadístico
}

\author{
Analysis of main elements in locking plating design \\ on a 2-part fracture of the surgical neck of the \\ humerus using FEM and statistical analysis
}

\author{
Ismael Mendoza-Muñoz ${ }^{1}$, Álvaro González-Ángeles ${ }^{1}$, \\ Gabriela Jacobo-Galicia ${ }^{1}$, Ana Castañeda ${ }^{1}$, \\ José Valenzuela-Gutiérrez ${ }^{1}$
}

\footnotetext{
${ }^{1}$ Universidad Autónoma de Baja California, Facultad de Ingeniería Mexicali. Blvd. Benito Juárez S/N, CP 21280, Mexicali, Baja California, México.

e-mail: ismael.mendoza@uabc.edu.mx, gangelesa@uabc.edu.mx, gabriela.jacobo@uabc.edu.mx, ana.castaneda@uabc.edu.mx, avalenzuela18@uabc.edu.mx
}

\section{RESUMEN}

Un gran número de fracturas de húmero proximal están siendo tratadas con osteosíntesis. Sin embargo, la pérdida de reducción sigue siendo un problema grave, lo que lleva al recorte de tornillo en la articulación humeral y la reoperación.

De acuerdo con la teoría del strain interfragmentario, se debe considerar el equilibrio entre una fijación flexible, que estimula la formación de callos, y una fijación inestable, que conduce al fallo del implante. Los principales elementos implicados en este caso son las condiciones fisiológicas de carga, la configuración de los tornillos de soporte, el material del implante y la calidad ósea. El objetivo de esta investigación es detectar la influencia de los elementos anteriores para formular una escena positiva que minimice el riesgo de fracaso.

Se utilizó un diseño de experimentos de tipo factorial en combinación con el uso del método de elementos finitos para dar lugar a la recogida de datos. Las condiciones de carga asemejan a: "sostener un peso muerto" (tensión), "levantar frontalmente un peso muerto" (flexión) y torsión.

Los resultados sugirieron que el material del implante es el elemento con mayor influencia y contribuye a estabilizar la fijación de la fractura, siguiendo la configuración de soporte. La práctica de incluir un soporte medial con tornillos calcar en diferentes calidades de tejido óseo ofrece una rigidez favorable que aquellos que no los utilizan. Esta afirmación es apoyada por resultados de informes publicados recientemente. Además, el soporte medial reduce el strain en la interfaz hueso-implante. Aunque estos resultados mostraron una evaluación demasiado moderada por el uso de un modelo isotrópico, son eficaces para conocer los elementos biomecánicos y su influencia para describir una tendencia de comportamiento y crear una base para futuros estudios. Además, el tiempo de cálculo disminuyó considerablemente.

Palabras clave: Biomecánica, fractura de húmero proximal, placas de bloqueo, tornillos de soporte medial.

\section{ABSTRACT}

A large number of proximal humerus fractures are being treated with osteosynthesis. However, loss of reduction remains a serious problem and it can cause screw cutout at the humeral joint and reoperation.

According to the interfragmentary strain theory, one should be aware of the balance between a flexible fixation, which stimulates callus formation, and an unstable fixation, leading to implant failure. The main elements that are implicated in this case include physiological loading conditions, support screws configuration, implant material and bone quality. The objective of this research was to detect the influence of the above elements to formulate a positive scene to reduce the risk of failure in the fracture.

A factorial experimental design was used in combination with the use of the finite element method to give 
rise to data collection.

The results suggested that implant material is the element with the most significant influence and it contributes to stabilizing the fracture fixation, following by the support configuration. The practice of including a medial support with calcar screws on different bone tissue qualities offers a more favorable rigidity than those who do not use them. This affirmation is supported by the results of recently published reports. In addition, the medial support reduces the strain in the bone-plate join. Although these results showed a too moderate evaluation per the use of an isotropic model, they are effective to know the biomechanical elements and their influence to describe a behavior trend and create a basis for future studies. In addition, the computing time decreased considerably.

Keywords: Biomechanics, humeral proximal fracture, locking plate, medial support screws.

\section{INTRODUCCIÓN}

La fractura de húmero proximal es una fractura común de la extremidad superior y representa aproximadamente el $5 \%$ de todas las fracturas [1,2]. Se espera que su incidencia aumente un $250 \%$ en los próximos 30 años, lo que tendrá un impacto considerable en los servicios de salud, ya que limitan gravemente la independencia de los pacientes en su vida diaria [3,4]. Esta fractura es una lesión típica del paciente mayor de 65 años de edad y debido a esto, la mayoría de estas fracturas están relacionadas con la osteoporosis [5,6]. Aunque la mayoría de estas reciben un tratamiento conservador, si presentan mala calidad ósea o conminución medial, el tratamiento quirúrgico se considera todo un desafío [2, 7, 8].

Estas fracturas se pueden clasificar de acuerdo al sistema utilizado por NEER [9]. Por ejemplo, señala que la fractura de 1-parte muestra un desplazamiento menor a $1 \mathrm{~cm}$ (desplazamiento mínimo) en uno de sus segmentos o angulado a menos de 45 grados independientemente del número de líneas de la fractura. La terminología de las fracturas desplazadas relaciona un patrón de desplazamiento (2-, 3- y 4-partes) y el segmento clave desplazado. Aunque se han reconocido otros tipos de fractura desde la introducción de la tomografía computarizada (TC), el sistema propuesto por Neer sigue siendo ampliamente utilizado porque no sólo constituye un esquema de clasificación descriptivo, sino que también explica el desplazamiento de cada fragmento basado en las fuerzas de tracción ejercidas por los músculos del manguito rotador.

Aproximadamente un $15 \%$ a $20 \%$ de todas las fracturas de humero proximal son desplazadas y tratadas quirúrgicamente de acuerdo con el sistema de Neer. El tratamiento a elegir en fracturas de 2-partes depende del grado de desplazamiento de la fractura, por ejemplo, las fracturas severas de 2-partes desplazadas se tratan normalmente quirúrgicamente. La Reducción Abierta y la Fijación Interna (RAFI) con placas de bloqueo es la opción de tratamiento más popular [10,11].

A pesar de que un gran número de fracturas de húmero proximal están siendo tratadas con osteosíntesis, continúa siendo un problema grave la pérdida de la reducción de los fragmentos desplazados, con tasas de colapso en una posición en varo que oscila entre un $15 \%$ a $40 \%$ [8,12]. En su revisión sistemática sobre las placas de bloqueo en estas fracturas, SPROUL et al. [13] identifican que la complicación anterior es de suma importancia porque puede conducir al recorte de tornillo en la articulación humeral y a la reoperación. Del mismo modo, CHA et al. [14] encontraron que son muy frecuentes estos padecimientos en los pacientes con una mala calidad ósea. Igualmente, existen complicaciones tempranas que incluyen: lesiones nerviosas o avasculares como lesiones del manguito rotador; y tardías, que comprenden necrosis avascular de la cabeza humeral, sepsis o no-unión. Estas últimas son generalmente las más graves y difíciles de tratar [15].

Se ha demostrado por medio del análisis biomecánico que la placa de bloqueo presenta una mayor estabilidad en contraste con la placa convencional o la fijación intramedular [8, 16, 17, 18, 19]. LILL et al. [20] por ejemplo, desarrollaron un estudio in vitro donde analizaron diferentes implantes no bloqueados y bloqueados bajo esfuerzos estáticos y dinámicos en varo. Se encontró que los implantes muy rígidos como la placa en $\mathrm{T}$ y el clavo proximal eran los más estables bajo ensayos estáticos pero mostraban el aflojamiento de los tornillos bajo carga dinámica. Por otro lado, el implante con una interfaz de placa de tornillo bloqueada mostraba mejores resultados en el anclaje óseo osteoporótico. De forma análoga, MOONOT et al. [21] añaden que en fracturas con mala calidad ósea, es recomendable el uso de tornillos de bloqueo bicorticales para aumentar la longitud de trabajo del tornillo y evitar un problema potencial en la interfaz entre la rosca del tornillo y el hueso. Asimismo, ZHANG et al. [12], llevaron a cabo un estudio en donde encontraron que el soporte de tornillo medial reduce la tendencia al colapso en varo en fracturas de tres y cuatro partes. Este último hallazgo es similar al recientemente reportado por LESCHEID et al. [22] donde demostraron la importancia bio-mecánica del soporte cortical medial con el uso de un modelo sintético de fractura humeral. De igual manera, GARDNER et al. [23] observaron que la restauración del calcar, ya sea a través de la reducción anatómica o con la colocación de tornillos a través del calcar cuando la trituración medular impide el 
contacto cortical, reduce significativamente la frecuencia del colapso del varo.

La hipótesis del strain interfragmentario predice que la curación de una la fractura ocurrirá sólo si el movimiento interfragmentario dividido por el ancho de la abertura de fractura es menor que el strain de fractura del hueso [24]. Esta hipótesis ha demostrado que la reducción anatómica no es necesaria para la curación del hueso ya que el strain medio (2\% a 10\%) puede promover la curación indirecta del hueso [25]. Los principios de una fijación flexible deben de emplearse cuando el objetivo es la curación indirecta con la formación de callos. Teniendo esto en cuenta, se debe de estar consciente del equilibrio entre una fijación flexible, que estimula la formación de callos y promueve el proceso de cicatrización, y una fijación inestable, que conduce a la no-unión y el fallo del implante. [26]. El uso de fijadores internos de bloqueo permite la formación de callos a través de una mayor flexibilidad en la estabilización de la fractura [24].

El presente estudio examinó los efectos que tiene diversos factores biomecánicos en la reducción abierta desde un enfoque estadístico, con el propósito de establecer los niveles óptimos de los mismos y con ello, disminuir las posibilidades de colapso en una posición en varo. Para la realización de esta investigación se adoptó un Diseño De Experimentos (DDE) de tipo factorial en combinación con el uso del Método de Elementos Finitos (MEF) para dar lugar a la recogida de datos. Este estudio ofrece algunas pistas importantes sobre el diseño de dispositivos de fijación en fracturas de húmero proximal, las cuales contribuyen a esta creciente área de investigación.

\section{MATERIALES Y MÉTODOS}

A continuación, se presenta información sobre el enfoque metodológico adoptado para este estudio, el cual hizo uso de una combinación de enfoques, al adoptar el Método de Elementos Finitos para la obtención de resultados bajo los principios biomecánicos que la literatura ofrece, como del uso de Diseño de Experimentos para el tratamiento e interpretación de resultados.

\subsection{Método de Elementos Finitos}

El MEF es la opción más versátil, adecuada y eficiente para el análisis de estructuras, debido a su versatilidad en el manejo de geometrías complejas y condiciones de frontera [27]. El uso del MEF ha sido fundamentalmente exitoso en articulaciones con una geometría relativamente sencilla y condiciones de carga bien definidas, como la cadera. Otras articulaciones que también se han estudiado ampliamente incluyen el hombro, la rodilla, y la espina dorsal $[28,29]$.

El método comúnmente utilizado en análisis biomecánicos mediante la aplicación MEF sigue el procedimiento descrito por MASIH et al. [30]. Se decidió que esta metodología proporciona una base más adecuada para la recogida de datos de este estudio. La Figura 1 presenta el procedimiento mencionado.

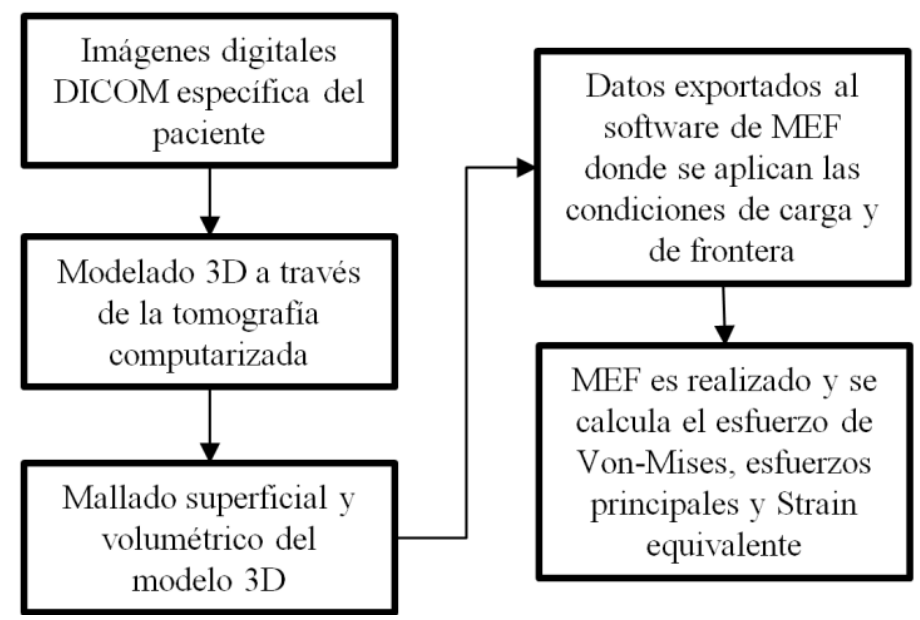

Figura 1: Procedimiento utilizado en análisis biomecánicos mediante la aplicación del MEF [30].

\subsection{Modelado 3D}

Con el fin de modelar el extremo proximal del húmero, se emplearon imágenes digitales DICOM pertenecientes al Laboratorio de Anatomía Humana y Embriología de la Universidad de Bruselas (ULB), Bélgica. Mediante el uso de un programa médico para la creación de modelos virtuales 3D (InVesalius 3.0) en conjunción con un programa de procesamiento y edición de imágenes (MeshLab), se logró obtener un modelo 
elemental, el cual es deseable para optimizar las etapas posteriores del análisis biomecánico mediante la aplicación del MEF. Este modelo se llevó a un programa de diseño mecánico (SolidWorks) donde finalmente, se modificó para el estudio de una fractura de 2-partes de cuello quirúrgico del húmero. Este tipo de fractura se seleccionó dado que es empleada frecuentemente en estudios biomecánicos [17, 31, 32, 33, 34, 35, 36]. La Figura 2 muestra la fractura antes mencionada, en donde la reducción y fijación es utilizando una placa de bloqueo.


Figura 2: Fractura de 2-partes de cuello quirúrgico de húmero la cual es reducida y fijada con placa de bloqueo [37].

Se eligió la placa de bloqueo PHILOS como referencia para el modelado del sistema de fijación empleado en el estudio, realizándose directamente en el programa de diseño mecánico (SolidWorks). Como sugiere SABALIC et al. [38], los elementos de fijación fueron modelados como cilindros lisos sin hilos con un diámetro de 3,5 mm.

Para estudiar el efecto biomecánico de la placa de bloqueo en una fractura de 2-partes de cuello quirúrgico del húmero, se propusieron dos Configuraciones de Soporte (CS): sin soporte medial y con soporte medial. La Figura 3 muestra estas CS mostrando tornillos al Calcar (C), tornillos Bicorticales (B) y tornillos de bloqueo de compresión localizados en la cabeza humeral (identificados con números). Basándonos en YANG et al. [39] se decidió ajustar la longitud de los tornillos en el ensamble, de tal forma que la punta del tornillo se situara a $2 \mathrm{~mm}$ dentro de la superficie de la cabeza humeral, permitiendo simular una excelente fijación del implante. Finalmente, es destinado un ensamble (modelo 3D) para cada CS, los cuales son analizados en el programa de análisis numérico (ANSYS Workbench 15.0).

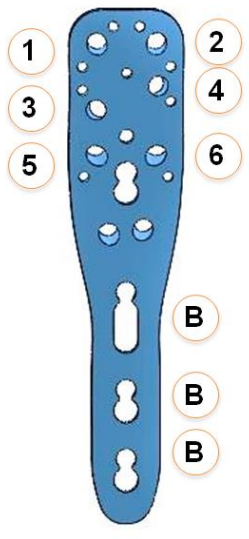

( I )

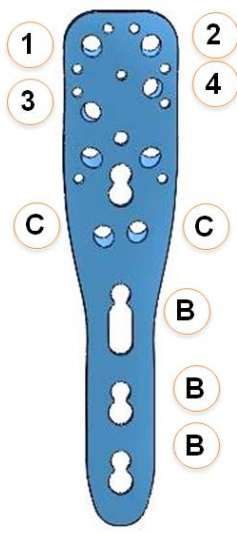

( II )

Figura 3: Niveles de Configuraciones de Soporte: (I) sin soporte medial y (II) con soporte medial. En la figura, los números muestran los tornillos de bloqueo de compresión, la letra "B" señalan los tornillos Bicorticales y la letra "C" presentan los tornillos al Calcar. 


\subsection{Mallado y propiedades de los materiales}

Los ensambles son mallados finamente con elementos cuadráticos (SOLID 187) empleando los detalles por defecto del programa de análisis numérico (ANSYS Workbench 15.0). Este tipo de elemento tiene un comportamiento de desplazamiento cuadrático y es adecuado para modelar mallas irregulares [40]. El mallado genera diferentes elementos y nodos para cada ensamble: 43572 elementos y 77548 nodos para el ensamble sin soporte medial y, 43951 elementos y 78938 nodos para el ensamble con soporte medial.

Es ampliamente aceptado considerar como isotrópicos el tejido cortical y esponjoso para caracterizar el comportamiento mecánico del hueso, aunque en realidad no lo son. Lo anterior permite simplificar el modelado del sistema, reduciendo considerablemente el tiempo de cómputo para la obtención de la respuesta a estudiar [29, 39, 41, 42, 43, 44, 45]. CLAVERT et al. [44] identificaron el módulo de elasticidad obtenido a través de la experimentación con tejido cortical y esponjoso del extremo superior del húmero para hueso sano y con osteoporosis, ambos considerados como materiales isotrópicos. La Tabla 1 presenta las propiedades mecánicas antes mencionadas, las cuales son adoptadas para la realización de esta investigación. Asimismo, BOGATAJ et al. [46] encontró que las pro-piedades mecánicas del tejido esponjoso mostraron un reducido efecto sobre los resultados de sus estudios biomecánicos debido a la gran diferencia en el módulo de elasticidad entre los tejidos cortical y esponjoso (de más de 20:1). Basándonos en la afirmación anterior se decidió no considerar el tejido esponjoso para el estudio.

Tabla 1: Propiedades mecánicas para hueso sano y con osteoporosis [44].

\begin{tabular}{l|l|l|l|l}
\hline \multirow{2}{*}{ PROPIEDAD } & \multicolumn{2}{|l|}{ HUESO CORTICAL } & \multicolumn{2}{l}{ HUESO TRABECULAR } \\
\cline { 2 - 5 } & Sano & Osteoporosis & Sano & Osteoporosis \\
\hline Módulo de Elasticidad & $16 \mathrm{GPa}$ & $12 \mathrm{GPa}$ & $550 \mathrm{MPa}$ & $250 \mathrm{MPa}$ \\
\hline Relación de Poisson & 0,3 & 0,3 & 0,3 & 0,3 \\
\hline
\end{tabular}

$\mathrm{L}$ as placas y tornillos de bloqueo de compresión están fabricados generalmente a base de acero inoxidable y titanio, ambos aceptados como materiales biocompatibles [24]. Las propiedades mecánicas de estos materiales fueron definidos por las fuentes de datos del programa de análisis numérico (ANSYS Workbench 15.0). La Tabla 2 presenta las propiedades antes mencionadas, tomando los materiales como elásticos e isotrópicos.

Tabla 2: Propiedades mecánicas del material para la osteosíntesis.

\begin{tabular}{l|l|l}
\hline PROPIEDAD & TITANIO & ACERO INOXIDABLE \\
\hline Módulo de Elasticidad & $96 \mathrm{GPa}$ & $193 \mathrm{GPa}$ \\
\hline Relación de Poisson & 0,36 & 0,31 \\
\hline
\end{tabular}

\subsection{Condiciones de carga y de frontera}

Las cargas fisiológicas que se ejercen en la extremidad superior son difíciles de definir debido a la compleja matriz de los músculos responsables del movimiento [21]. Sin embargo, se ha publicado una considerable cantidad de evidencia sobre diferentes procedimientos utilizados para estimar las cargas fisiológicas presentadas durante distintas actividades diarias y particularmente en la posición de "caer sobre un brazo estirado", siendo esta última, ampliamente aceptada al aplicar una carga puntual de compresión vertical en la cabeza humeral [47,48]. En el presente, muy pocos estudios han examinado el desempeño biomecánico de los sistemas de fijación bajo cargas que representen la posición de "sostener un peso muerto". En la Figura 4(a) se muestra la posición antes descrita. Esta investigación busca analizar la condición antes descrita, al aplicar una carga puntal de tensión en la cabeza humeral. Se ha decidido elegir una magnitud de carga de $50-150 \mathrm{~N}$ en base a los estudios realizados por ZHANG et al. [48]. Adicionalmente, se propone estudiar el sistema de fijación considerando tareas que resultan en flexión (Figura 4(b)) y torsión (Figura 4(c)) del brazo. Un evento frecuente que podría ocasionar la flexión del brazo se presenta al "levantar frontalmente un peso muerto" al extender el brazo. Se propone utilizar una carga de $10 \mathrm{~N}$ en la cabeza humeral. El movimiento que podría asemejar la condición de torsión del brazo afectando directamente la región proximal, no puede considerarse como un movimiento "natural", sin embargo, se propone un par de torsión de 10Nm en la cabeza humeral a 
fin de evaluar el sistema de fijación bajo esta condición. Para todos los anteriores, se propone fijar el extremo opuesto a la cabeza humeral, siendo la parte inferior del modelo.

Como argumentan INZANA et al. [49] y SABALIC et al. [38], es oportuno utilizar un coeficiente de fricción de 0,3 en la interfaz de hueso-implante. ZHANG et al. [48] sostiene usar restricciones de movimiento en la interfaz de tornillo-hueso como en la interfaz placa-tornillo, simulando el comportamiento de un cierre ajustado.

\subsection{Diseño de Experimentos}

El DDE es una técnica poderosa utilizada tanto para estudiar nuevos procesos como para conocer más acerca de un proceso existente, buscando su optimización [50].

En el MEF, es común estimar una o más variables de respuestas (esfuerzo de Von-Mises, esfuerzos principales, etc.). Las propiedades del material, la creación de un modelo 3D como las condiciones de carga y de frontera se utilizan como factores y reciben valores discretos (niveles) que se dividen equitativamente por el rango de cada factor. Se pueden incorporar todos los factores que pudieran tener un efecto sobre la respuesta del sistema [51]. La conclusión de un experimento está determinada por una suma de factores. En un Diseño Factorial (DF) se permite estimar el efecto de estos factores y sus interacciones con un número mínimo de experimentos [52].
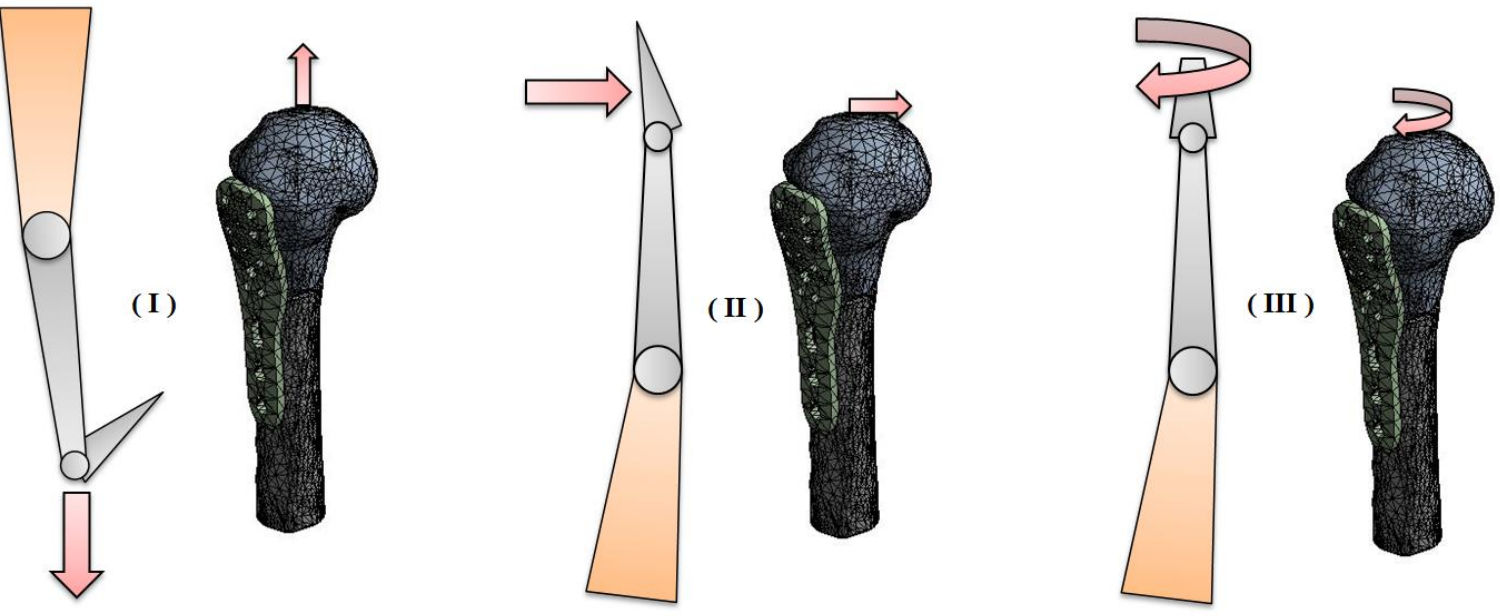

Figura 4: Condiciones de carga en posición de: (I) "sostener un peso muerto", (II) "levantar frontalmente un peso muerto" y, (III) torsión.

Para evaluar los efectos de los factores discutidos para el caso de "sostener un peso muerto", se utilizó un modelo de DF de dos niveles $2^{\wedge} 4$ con pseudo-puntos centrales para cada factor cuantitativo. Considerando factores tanto cuantitativos (carga) como cualitativos (configuración de soporte, tipo de hueso y material de placa). Se tiene que el experimento está formado por 24 corridas en total. La Tabla 3 muestra los factores como niveles utilizados para el modelo del DF propuesto.

Tabla 3: Factores como niveles usados para DF de dos niveles $2^{\wedge} 4$ con pseudo-puntos centrales.

\begin{tabular}{|c|c|c|c|}
\hline \multirow[t]{2}{*}{ FACTOR } & \multicolumn{3}{|l|}{ NIVEL } \\
\hline & - & Pseudo-punto & + \\
\hline A: Configuración de Soporte & Sin soporte medial & $\mathrm{n} / \mathrm{a}$ & Con soporte medial \\
\hline B: Tipo de Hueso & Osteoporosis & $\mathrm{n} / \mathrm{a}$ & Sano \\
\hline C: Material de Placa & Titanio & $\mathrm{n} / \mathrm{a}$ & Acero Inoxidable \\
\hline D: Carga (N) & 50 & 100 & 150 \\
\hline
\end{tabular}

En las condiciones de "levantar frontalmente un peso muerto" y torsión, se propuso un modelo de DF fraccionado de dos niveles $2^{\wedge}(3-1)$. Los factores a estudiar son únicamente de tipo cualitativos (configuración de soporte, tipo de hueso y material de placa) debido a la propuesta de carga mencionada antes, siendo los 3 
primeros que se muestran en la Tabla 3. Se tiene que cada experimento está formado por 4 corridas en total.

\section{RESULTADOS}

\subsection{Posición de "sostener un peso muerto"}

Para el propósito del análisis, se seleccionó como respuesta de sistema la deformación total y el esfuerzo de von Mises máximo. La deformación total máxima fue elegida ya que es aplicable calcular el strain interfragmentario de cada corrida y determinar el nivel de fijación (flexible o inestable) en la fractura. Igualmente, para explorar como cada modelo resiste o falla de acuerdo las condiciones propuestas, el esfuerzo de von Mises es una respuesta del sistema utilizada ampliamente para cuantificar dicho evento. Es importante señalar que de acuerdo a literatura existente sobre la interacción que existe entre húmero fracturado como de la placa de bloqueo, las respuestas a estudiar corresponden a los modelos 3D del ambos al ser ensamblados. Esto implica que se desarrollaron $4 \mathrm{DF}$ de dos niveles $2^{\wedge} 4$ con pseudo-puntos centrales, necesitando de 96 corridas en total para completar el estudio. La Tabla 4 presenta los valores promedio obtenidos para cada combinación (excluyendo el factor Carga).

Tabla 4: Valores promedio obtenidos para cada respuesta del sistema (hueso fracturado y placa de bloqueo) incluyendo strain interfragmentario como rigidez en la fractura en posición de "sostener un peso muerto".

\begin{tabular}{|c|c|c|c|c|c|c|c|c|c|c|}
\hline \multirow[t]{2}{*}{ No. } & \multicolumn{4}{|c|}{ FACTOR } & \multicolumn{3}{|c|}{ EN HUESO FRACTURADO } & \multicolumn{2}{|c|}{ EN PLACA DE BLOQUEO } & \multirow{2}{*}{$\begin{array}{l}\text { Rigidez en la } \\
\text { fractura } \\
(\mathrm{N} / \mathrm{mm})\end{array}$} \\
\hline & & $T_{0}$ & ${ }_{0}$ & I & $\begin{array}{l}\text { Deform. Total } \\
\text { máx. }(\mathrm{mm})\end{array}$ & \begin{tabular}{|l} 
Strain \\
$(\%)$
\end{tabular} & $\begin{array}{l}\text { Esfuerzo } \\
\text { máx. (MPa) }\end{array}$ & $\begin{array}{l}\text { Deform. Total } \\
\text { máx. }(\mathrm{mm})\end{array}$ & $\begin{array}{l}\text { Esfuerzo } \\
\text { máx. (MPa) }\end{array}$ & \\
\hline 1 & $+\mathrm{A}$ & $+\mathrm{B}$ & $+\mathrm{C}$ & - & 0.55 & 5.33 & 12.28 & 0.42 & 42.00 & 184.38 \\
\hline 2 & $+\mathrm{A}$ & $+\mathrm{B}$ & $-\mathrm{C}$ & - & 0.89 & 8.67 & 12.50 & 0.68 & 67.67 & 177.39 \\
\hline 3 & $+\mathrm{A}$ & $-\mathrm{B}$ & $+\mathrm{C}$ & - & 0.57 & 5.67 & 11.38 & 0.44 & 43.67 & 184.40 \\
\hline 4 & $+\mathrm{A}$ & $-\mathrm{B}$ & $-\mathrm{C}$ & - & 0.91 & 9.33 & 11.71 & 0.70 & 69.67 & 177.40 \\
\hline 5 & $-\mathrm{A}$ & $+\mathrm{B}$ & $+\mathrm{C}$ & - & 0.66 & 6.67 & 15.76 & 0.46 & 46.00 & 166.46 \\
\hline 6 & $-\mathrm{A}$ & $+\mathrm{B}$ & $-\mathrm{C}$ & - & 1.12 & 11.33 & 15.44 & 0.79 & 78.67 & 164.94 \\
\hline 7 & $-\mathrm{A}$ & $-B$ & $+\mathrm{C}$ & - & 0.68 & 6.67 & 13.79 & 0.48 & 48.00 & 166.36 \\
\hline 8 & $-\mathrm{A}$ & $-\mathrm{B}$ & $-\mathrm{C}$ & - & 1.15 & 11.67 & 14.05 & 0.80 & 80.00 & 164.86 \\
\hline
\end{tabular}

El strain interfragmentario fue calculado como se muestra en la siguiente fórmula:

$\epsilon=\Delta L / L$

Donde:

$\epsilon=$ Strain

$\Delta L=$ Desplazamiento

$L=$ Logitud inicial

Además, la rigidez en la fractura fue incorporada en la Tabla 3 y se calculó como sigue:

$K=F / \Delta L$

Donde:

$K=$ Rigidez

$\mathrm{F}=$ Fuerza aplicada

$\Delta L=$ Desplazamiento

Un programa estadístico (Minitab 17) fue utilizado para desarrollar los DF mencionados, resultando en análisis de varianza (ANOVA), revisión de residuales, identificación de efectos estandarizados, además de la 
identificación de los efectos principales e interacciones. Se ha asegurado que se cumple con los supuestos estadísticos tomando como referencia las gráficas de residuales. En las Figuras 5, 6, y 7 se muestran el gráfico de Pareto de efectos estandarizados, así como los efectos principales y las gráficas de interacción para cada variable de respuesta en los modelos estudiados (hueso fracturado y placa de bloqueo). El grafico de Pareto de efectos estandarizados nos permite visualizar cuales efectos tienen un mayor impacto sobre la respuesta del sistema. Este representa los efectos divididos entre su error estándar. Por otra parte, un efecto principal es igual a la respuesta promedio observada en el nivel alto de un factor, menos la respuesta promedio en el nivel bajo. Finalmente, se dice que dos factores interactúan entre sí o tienen un efecto de interacción sobre la variable de respuesta, cuando el efecto de un factor depende del nivel en que se encuentra el otro [53].

Con el fin de comprender como cada uno de los factores propuestos actúan sobre la fijación de la fractura analizada, es importante tener primeramente en claro el rol del factor D (Carga) en el estudio. Como ya se mencionó en enunciados anteriores, el factor $\mathrm{D}$ es una entrada del sistema observado, pero además, es un estímulo indispensable para el experimento ya que sin una carga aplicada al sistema, no se tendría ninguna respuesta debido a la naturaleza del mismo. Desde otro punto de vista, el factor D describe las condiciones de operación de la fractura estudiada, siendo ajeno al mismo sistema de fijación examinado. Por lo tanto, se propone suprimir el factor D como significante.



( I)



( III )

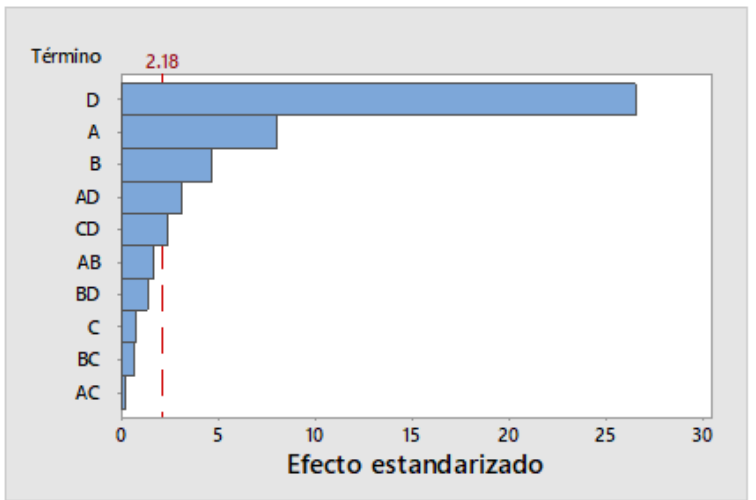

( II )



(IV)

Figura 5: Gráficos de Pareto de efectos estandarizados para cada respuesta de sistema en posición de "sostener un peso muerto"; (I) deformación total en hueso, (II) esfuerzo de von Mises en hueso, (III) deformación total en placa y (IV) esfuerzo de von Mises en placa.

La Tabla 5 proporciona una visión general de resultados mostrados en los gráficos de Pareto de efectos estandarizados para cada respuesta de sistema (Figura 5). En la Tabla 5 se enumeran los factores que son estadísticamente significativos, desde el efecto más grande hasta el efecto más pequeño. Se distingue con * el primer resultado ya que corresponde al factor D (Carga), el cual se omite para interpretación. 
Tabla 5: Factores estadísticamente significativos de acuerdo a los gráficos de Pareto de efectos estandarizados en posición de "sostener un peso muerto".

\begin{tabular}{l|l|l|l|l}
\hline GRADO DE & EN HUESO FRACTURADO & \multicolumn{2}{|l}{ EN PLACA DE BLOQUEO } \\
\cline { 2 - 5 } SIGNIFICANCIA & $\begin{array}{l}\text { Deformación } \\
\text { total máx. }\end{array}$ & Esfuerzo máx. & $\begin{array}{l}\text { Deformación } \\
\text { total máx. }\end{array}$ & Esfuerzo máx. \\
\hline $1^{*}$ & $\mathrm{D}^{*}$ & $\mathrm{D}^{*}$ & $\mathrm{D}$ & $\mathrm{D}$ \\
\hline 2 & $\mathrm{C}$ & $\mathrm{A}$ & $\mathrm{C}$ & $\mathrm{A}$ \\
\hline 3 & $\mathrm{~A}$ & $\mathrm{~B}$ & $\mathrm{~A}$ & $\mathrm{C}$ \\
\hline 4 & $\mathrm{~B}$ & $\mathrm{AD}$ & $\mathrm{B}$ & $\mathrm{AC}$ \\
\hline 5 & $\mathrm{AC}$ & $\mathrm{CD}$ & $\mathrm{AC}$ & $\mathrm{AD}$ \\
\hline
\end{tabular}

Los resultados de la Tabla 5 muestran que comparten los mismos factores estadísticamente significativos en la deformación total máxima en hueso fracturado como en placa de bloqueo, de los cuales destacan: factor C (Material de Placa), factor A (Configuración de Soporte), factor B (Tipo de Hueso) e interacción AC. A partir de la Figura 6 podemos definir las mejores condiciones para proporcionar un nivel de fijación que promueva la curación del hueso, tomando como referencia la hipótesis de strain interfragmentario. Lo anterior se satisface al reducir esta respuesta para ambos sistemas, sugiriendo seleccionar los niveles en cada factor e interacción: $+\mathrm{C}$ (acero inoxidable), $+\mathrm{A}$ (con soporte medial), $+\mathrm{B}$ (hueso sano) y $+\mathrm{A}+\mathrm{C}$ (con soporte medial y acero inoxidable).

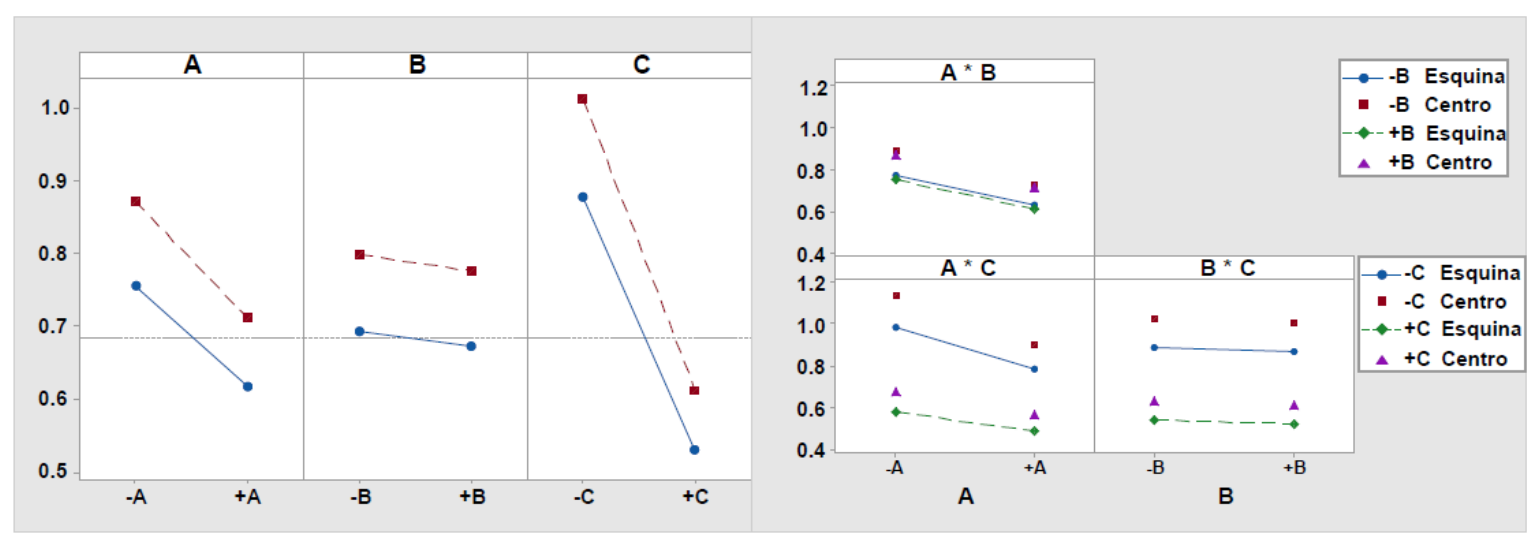

( I )

( II )

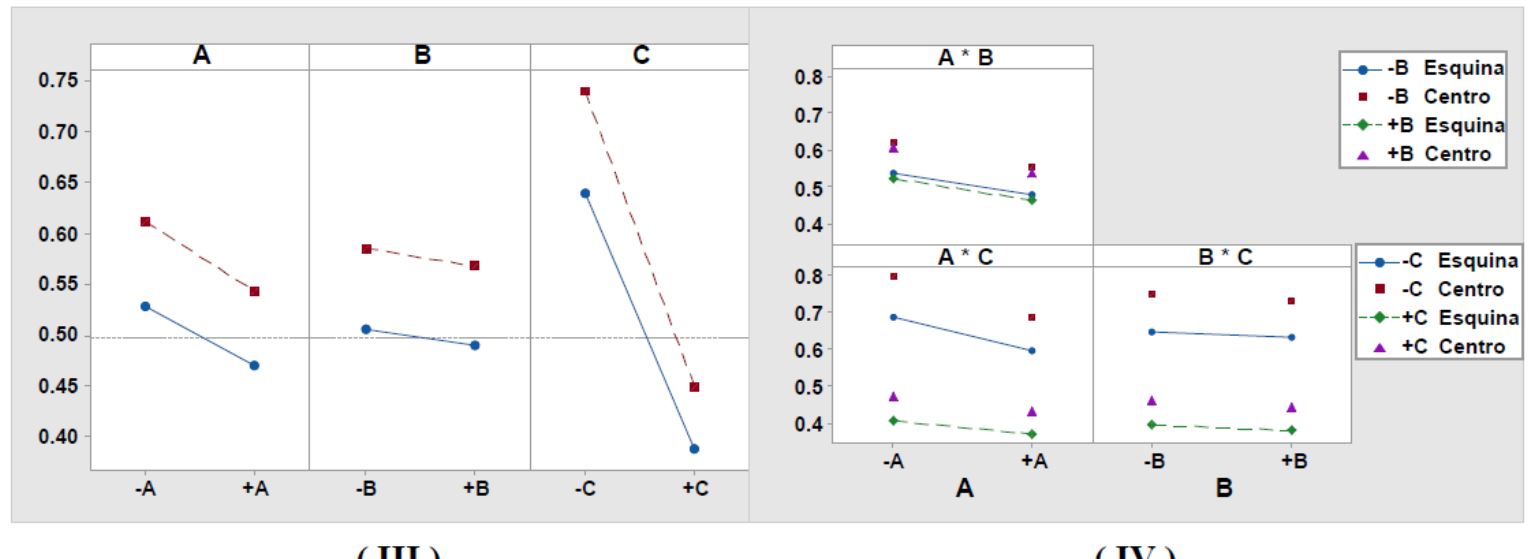

( III )

( IV)

Figura 6: Gráficos de efectos principales e interacción para la deformación total máxima en hueso fracturado (I y II) y en placa de bloqueo (III y IV) en posición de "sostener un peso muerto".

Como se puede observar en la Figura 7, ambos sistemas (hueso fractura y placa de bloqueo) presentan sus respectivos factores estadísticamente significativos para el esfuerzo de von Mises máximo. Al igual que la deformación total máxima, se pretende obtener valores mínimos para disminuir la posibilidad de fallo del 
sistema de fijación estudiado. Tomando como referencia adicional la Tabla 5, de tiene que para el hueso fracturado, el factor A (Configuración de Soporte), factor B (Tipo de Hueso) interacción AD e interacción CD son estadísticamente significativos. Los niveles que ofrecen disminuir esta respuesta son: +A (con soporte medial) y -B (hueso con osteoporosis). Por el contrario, el factor A (Configuración de Soporte), factor C (Material de Placa), interacción AC e interacción AD se presentan como estadísticamente significativos en la placa de bloqueo. Se propone elegir los niveles $-\mathrm{A}$ ( $\sin$ soporte medial), $-\mathrm{C}$ (titanio) y $-\mathrm{A}-\mathrm{C}$ (sin soporte medial y titanio) para minimizar la respuesta.

En referencia con la hipótesis de strain interfragmentario, la Tabla 4 revela que las combinaciones de niveles en cada factor que no cumple con un strain medio ( $2 \%$ a $10 \%)$ son: $-\mathrm{A}$ (sin soporte medial) $-\mathrm{B}$ (hueso osteoporótico) y - C (titanio), así como también la combinación -A (sin soporte medial), +B (hueso sano) y -C (titanio). Lo anterior podría definirse como el sistema de fijación que conduce a la no-unión y fallo del implante debido a su inestabilidad, demostrando que el factor C (Material en Placa) es decisivo para establecer una fijación flexible.

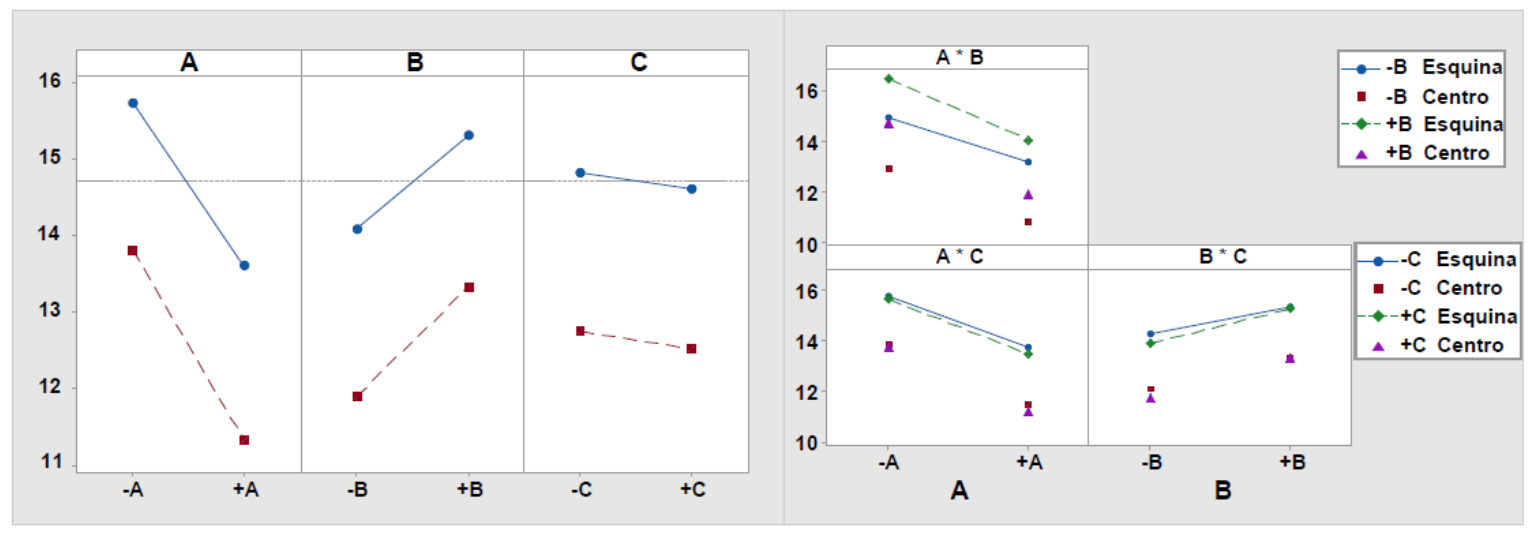

( I )

( II )

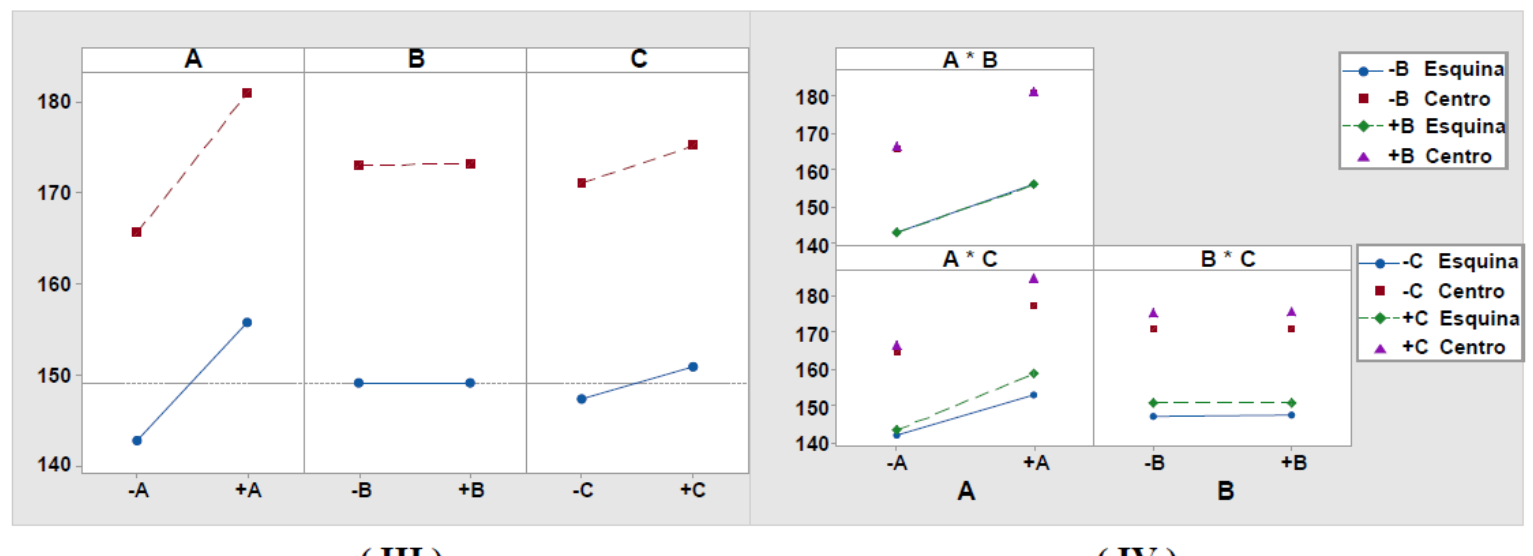

( III )

( IV )

Figura 7: Gráficos de efectos principales e interacción para el esfuerzo de von Mises máximo en hueso fracturado (I y II) y en placa de bloqueo (III y IV) en posición de "sostener un peso muerto".

La Figura 8 presenta el gráfico Carga deformación total en el hueso fracturado, la cual es construida tomando como referencia los datos de la Tabla 4 para la rigidez en la fractura calculados a partir de la Fórmula (2). A partir de este gráfico, se puede observar que no solamente el factor C (Material en Placa) es concluyente para lograr una fijación flexible, sino también el factor A (Configuración de Soporte) juega un papel importante. Se observa que nivel $+\mathrm{A}$ (con soporte medial) ofrece una mayor rigidez en la fractura en ambos niveles del factor $\mathrm{C}$ (titanio y acero inoxidable). 


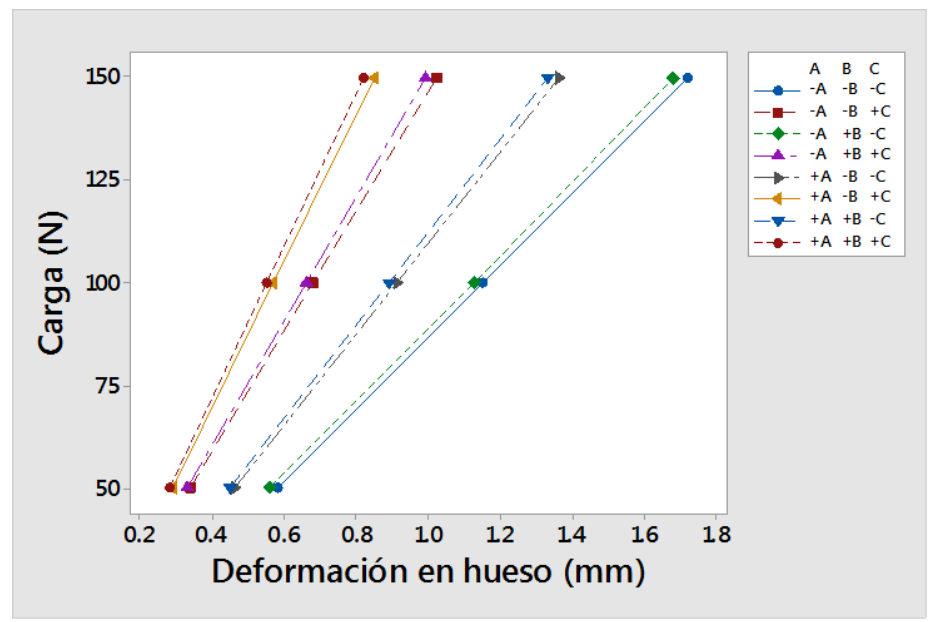

Figura 8: Gráfico carga - deformación total en el hueso fracturado en posición de "sostener un peso muerto".

La Figura 9 muestra la distribución del esfuerzo de von Mises máximo en placa de bloqueo para ambas configuraciones de soporte. Se concluye que el esfuerzo de von Mises se encuentra distribuido en zonas mayormente uniformes en la osteosíntesis sin soporte medial (-A) en comparación con su homóloga. Sin embargo, la placa de bloqueo con soporte medial $(+\mathrm{A})$ presenta regiones con valores menores de esfuerzo, lo que la hace ser la mejor alternativa desde esta respuesta.
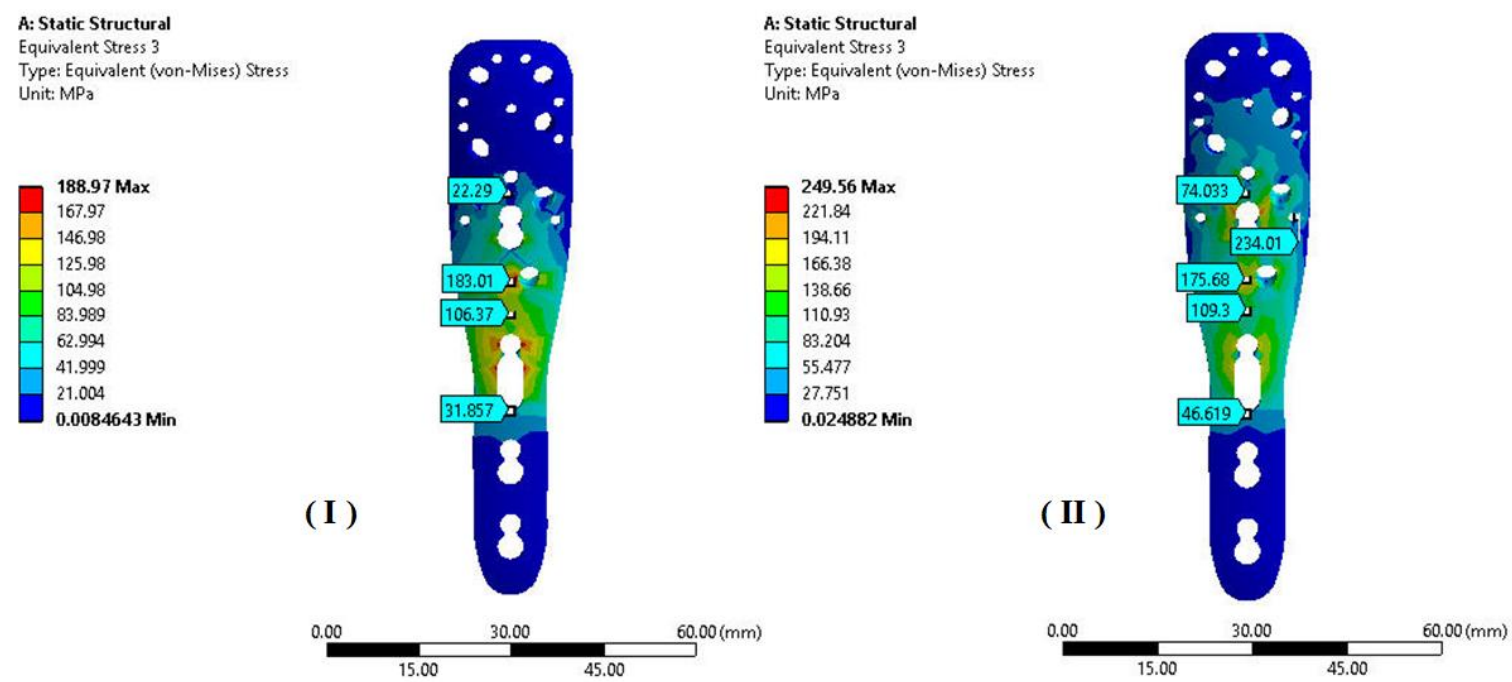

Figura 9: Distribución del esfuerzo de von Mises máximo en placa de bloqueo; (I) con soporte medial +A y (II) sin soporte medial -A, ambos en posición de "sostener un peso muerto".

\subsection{Posición de "levantar frontalmente un peso muerto"}

La Tabla 6 presenta los valores obtenidos bajo la posición de "levantar frontalmente un peso muerto". Con los datos mostrados en la Tabla anterior, se pudo desarrollar los 4 DF fraccionados de dos niveles $2^{\wedge}(3-1)$ para cada variable de respuesta en los modelos estudiados (hueso fracturado y placa de bloqueo). 
Tabla 6: Valores obtenidos para cada respuesta del sistema (hueso fracturado y placa de bloqueo) incluyendo strain interfragmentario como rigidez en la fractura en posición de "levantar frontalmente un peso muerto".

\begin{tabular}{|c|c|c|c|c|c|c|c|}
\hline \multirow[t]{3}{*}{ No. } & \multirow{2}{*}{\multicolumn{3}{|c|}{ FACTOR }} & \multicolumn{2}{|c|}{ EN HUESO FRACTURADO } & \multicolumn{2}{|c|}{ EN PLACA DE BLOQUEO } \\
\hline & & & & \multirow{2}{*}{$\begin{array}{l}\text { Deform. Total } \\
\text { máx. }(\mathrm{mm})\end{array}$} & \multirow{2}{*}{$\begin{array}{l}\text { Esfuerzo } \\
\text { máx. (MPa) }\end{array}$} & \multirow{2}{*}{$\begin{array}{l}\text { Deform. Total } \\
\text { máx. (mm) }\end{array}$} & \multirow{2}{*}{$\begin{array}{l}\text { Esfuerzo } \\
\text { máx. (MPa) }\end{array}$} \\
\hline & A & B & $\mathrm{C}$ & & & & \\
\hline 1 & $-\mathrm{A}$ & $-\mathrm{B}$ & $-\mathrm{C}$ & 0.22 & 7.55 & 0.14 & 14.32 \\
\hline 2 & $+\mathrm{A}$ & $-\mathrm{B}$ & $-\mathrm{C}$ & 0.28 & 6.81 & 0.18 & 14.57 \\
\hline 3 & $-\mathrm{A}$ & $+\mathrm{B}$ & $-\mathrm{C}$ & 0.28 & 11.12 & 0.17 & 43.82 \\
\hline 4 & $+\mathrm{A}$ & $+\mathrm{B}$ & $-\mathrm{C}$ & 0.29 & 11.99 & 0.16 & 46.55 \\
\hline
\end{tabular}

En la Figura 10 se muestra el gráfico de Pareto de efectos estandarizados, en donde aprecia que solamente el factor B (Tipo de Hueso) es estadísticamente significativo para el esfuerzo de von Mises máximo en la placa de bloqueo. En contraste, se tiene que para variables de respuesta restantes, los factores estudiados (Configuración de Soporte, Tipo de Hueso y Material de Placa) no tienen un mayor impacto sobre las mismas.

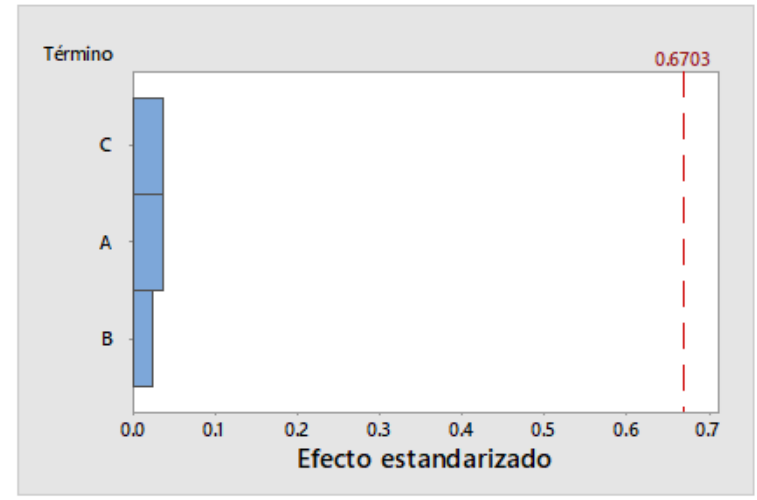

( I)



( III )



( II )

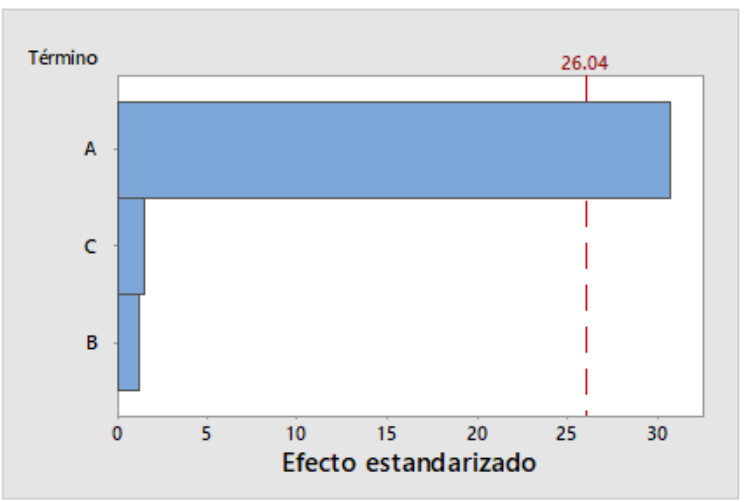

(IV)

Figura 10: Gráficos de Pareto de efectos estandarizados para cada respuesta de sistema en posición de "levantar frontalmente un peso muerto"; (I) deformación total en hueso, (II) esfuerzo de von Mises en hueso, (III) deformación total en placa y (IV) esfuerzo de von Mises en placa.

El grafico de efectos principales para el esfuerzo de von Mises máximo en la placa de bloqueo es mostrado en la Figura 11. Tomando como referencia lo mencionado anteriormente, se sugiere que el nivel $+\mathrm{A}$ (con soporte medial) lograría obtener valores mínimos de esfuerzo en la placa de bloqueo. 


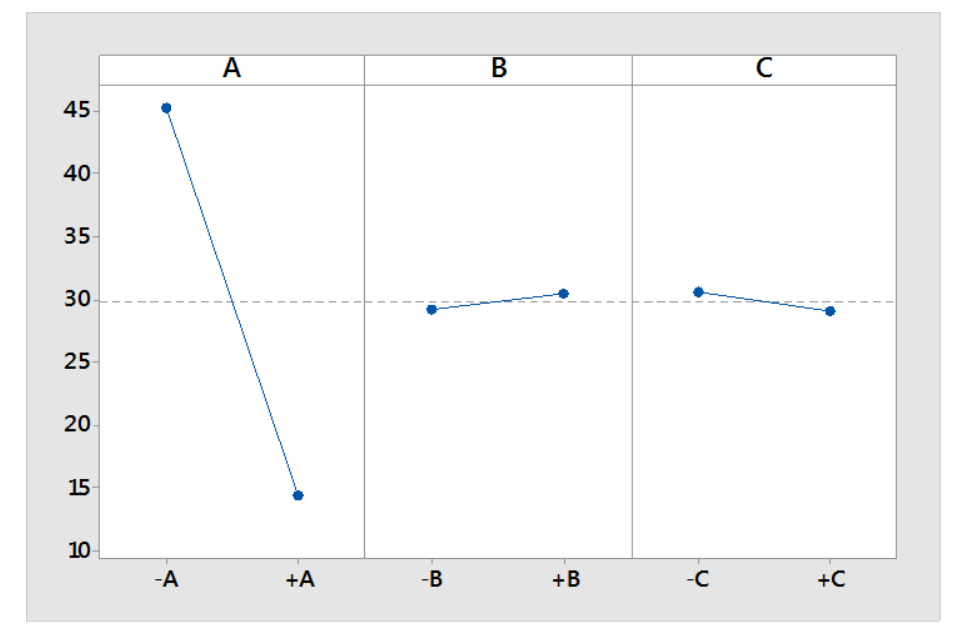

Figura 11: Gráficos de efectos principales para el esfuerzo de von Mises máximo para placa de bloqueo en posición de "levantar frontalmente un peso muerto".

\subsection{Posición de torsión}

La Tabla 7 muestra los valores obtenidos bajo la posición de torsión. Con los datos mostrados en la Tabla anterior, se pudo desarrollar los $4 \mathrm{DF}$ fraccionados de dos niveles $2^{\wedge}(3-1)$ para cada variable de respuesta en los modelos estudiados (hueso fracturado y placa de bloqueo).

Tabla 7: Valores obtenidos para cada respuesta del sistema (hueso fracturado y placa de bloqueo) incluyendo strain interfragmentario como rigidez en la fractura en torsión.

\begin{tabular}{|c|c|c|c|c|c|c|c|}
\hline \multirow[t]{3}{*}{ NO. } & \multicolumn{3}{|c|}{ FACTOR } & \multicolumn{2}{|c|}{ EN HUESO FRACTURADO } & \multicolumn{2}{|c|}{ EN PLACA DE BLOQUEO } \\
\hline & & & & \multirow{2}{*}{$\begin{array}{l}\text { Deform. Total } \\
\text { máx. }(\mathrm{mm})\end{array}$} & \multirow{2}{*}{$\begin{array}{l}\text { Esfuerzo } \\
\text { máx. (MPa) }\end{array}$} & \multirow{2}{*}{$\begin{array}{l}\text { Deform. Total } \\
\text { máx. }(\mathrm{mm})\end{array}$} & \multirow{2}{*}{$\begin{array}{l}\text { Esfuerzo } \\
\text { máx. (MPa) }\end{array}$} \\
\hline & A & B & C & & & & \\
\hline 1 & $-\mathrm{A}$ & $-\mathrm{B}$ & $-\mathrm{C}$ & 3.83 & 316.65 & 1.44 & 678.47 \\
\hline 2 & $+\mathrm{A}$ & $-\mathrm{B}$ & $-\mathrm{C}$ & 5 & 31 & 1.91 & 604.24 \\
\hline 3 & $-\mathrm{A}$ & $+\mathrm{B}$ & $-\mathrm{C}$ & 4.94 & 451 & 1.72 & 722.34 \\
\hline 4 & $+\mathrm{A}$ & $+\mathrm{B}$ & $-\mathrm{C}$ & 6.49 & 451.08 & 2.08 & 643.93 \\
\hline
\end{tabular}

En la Figura 12 se presenta el gráfico de Pareto de efectos estandarizados, en donde se aprecia que únicamente el factor B (Tipo de Hueso) es estadísticamente significativo para el esfuerzo de von Mises máximo en el hueso fracturado. Por otro lado, se tiene que para variables de respuesta restantes, los factores propuestos (Configuración de Soporte, Tipo de Hueso y Material de Placa) no tienen un mayor impacto sobre las mismas. 




( I )

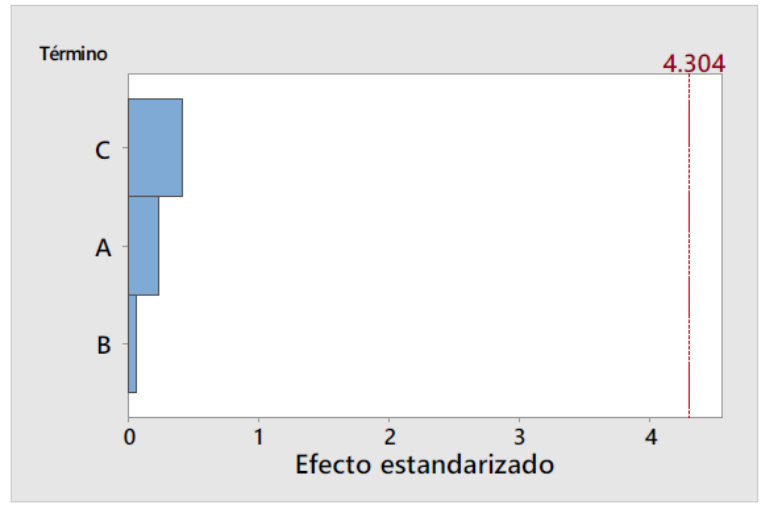

( III )

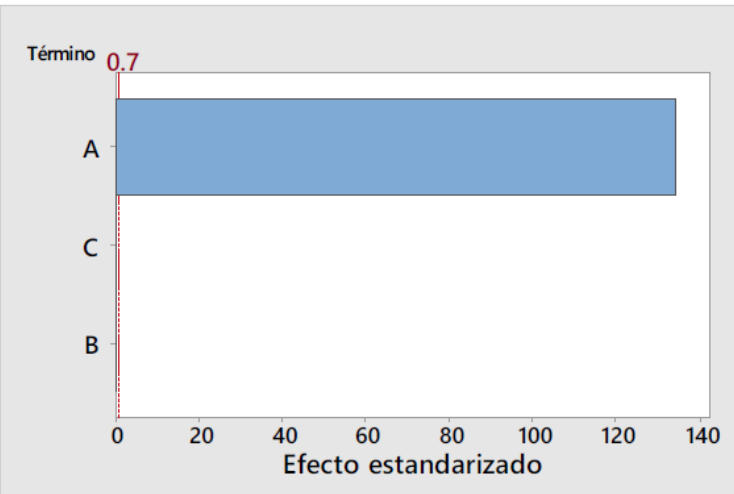

( II )

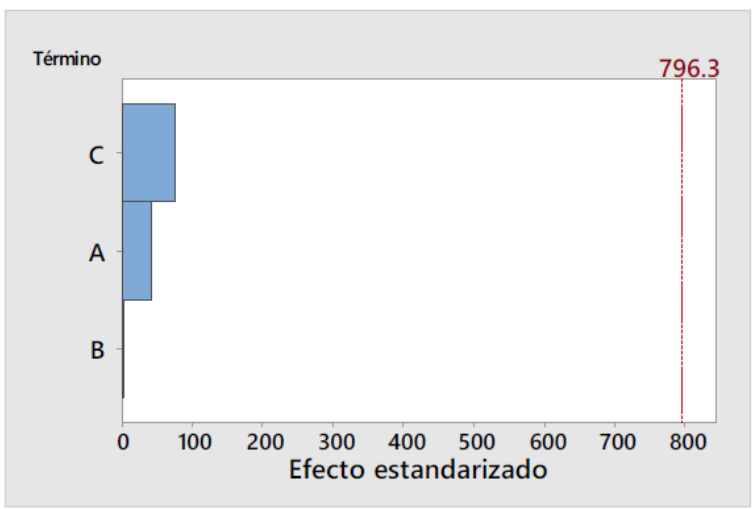

( IV )

Figura 12: Gráficos de Pareto de efectos estandarizados para cada respuesta de sistema en torsión; (I) deformación total en hueso, (II) esfuerzo de von Mises en hueso, (III) deformación total en placa y (IV) esfuerzo de von Mises en placa.

El grafico de efectos principales para el esfuerzo de von Mises máximo en el hueso fracturado es presentado en la Figura 13. Al tomar referencia lo mencionado en el párrafo anterior, se propone que el nivel $+\mathrm{A}$ (con soporte medial) mostraría valores mínimos de esfuerzo en el hueso fracturado.

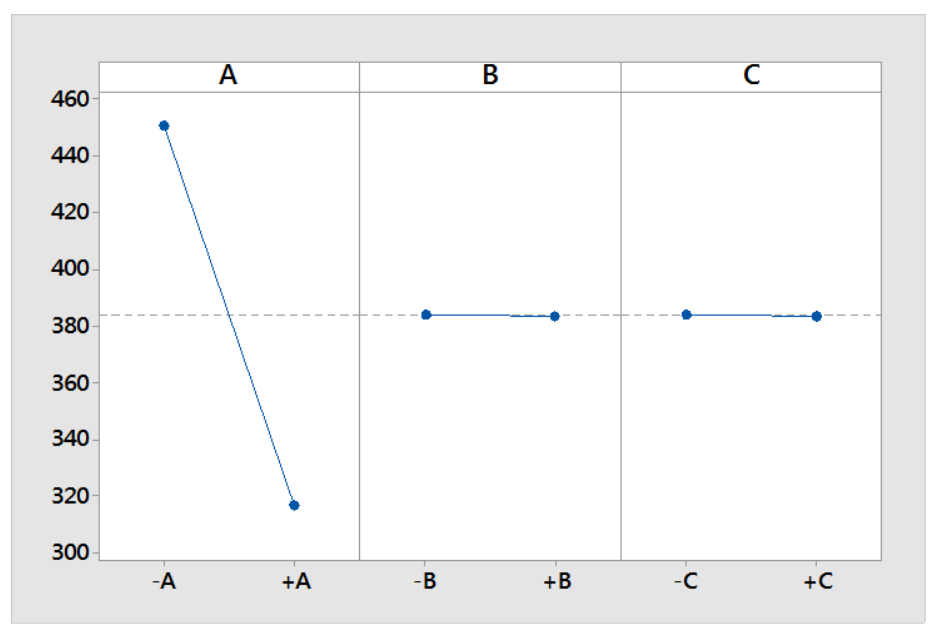

Figura 13: Gráficos de efectos principales para el esfuerzo de von Mises máximo para hueso fracturado en torsión. 


\section{DISCUSIÓN}

Una estructura preliminar para cada respuesta del sistema en el caso de "sostener un peso muerto" es presentada en la Tabla 8, tomando como referencia la información mostrada en las Figuras 5 a 8 así como conclusiones preliminares. De esta Tabla se desprende que el escenario más favorable para la fractura de 2-partes del cuello quirúrgico del húmero se encuentra considerando: soporte medial $(+\mathrm{A})$, tejido óseo sano $(+\mathrm{B})$ y acero inoxidable $(+\mathrm{C})$.

Tabla 8: Factores estadísticamente significativos de acuerdo a los gráficos de Pareto de efectos estandarizados para "sostener un peso muerto".

\begin{tabular}{l|l|l|l|l|l}
\hline SISTEMA & RESPUESTA & A & B & C & D \\
\hline \multirow{2}{*}{ Hueso fracturado } & Deform. Total máx. & $+\mathrm{A}$ & $+\mathrm{B}$ & $+\mathrm{C}$ & $\mathrm{D}$ \\
\cline { 2 - 6 } & Esfuerzo máx. & $+\mathrm{A}$ & $+\mathrm{B}$ & $+\mathrm{C}$ & $\mathrm{D}$ \\
\hline \multirow{2}{*}{ Placa de bloqueo } & Deform. Total máx. & $+\mathrm{A}$ & $-\mathrm{B}$ & $+\mathrm{C}$ & $\mathrm{D}$ \\
\cline { 2 - 6 } & Esfuerzo máx. & $-\mathrm{A}$ & $\mathrm{B}$ & $-\mathrm{C}$ & $\mathrm{D}$ \\
\hline Escenario más favorable & $+\mathrm{A}$ & $+\mathrm{B}$ & $+\mathrm{C}$ & $\mathrm{D}$ \\
\hline
\end{tabular}

Estos resultados confirman el argumento de usar un soporte medial (+A) como la mejor estrategia de éxito en este tipo de fractura, al minorar los valores de strain interfragmentario al igual que el esfuerzo en la interfaz implante-hueso, los cuales impulsan la formación de callo como el proceso de cicatrización, logrando reducir el riesgo de fallo del implante. A pesar de que en la Tabla 8 se observa una discrepancia en el esfuerzo de von Mises máximo para placa de bloqueo, se presenta un incremento del 7.5\% (de $166.5 \mathrm{MPa}$ a 184.4 $\mathrm{MPa}$ ) al sugerir dicha propuesta, lo cual puede ser insustancial para la resistencia mecánica del material de la osteosíntesis. Además, es importante tener en cuenta que el uso del soporte medial (+A) brinda una mayor rigidez en la fractura, el cual tiene una gran influencia sobre el strain interfragmentario lo que respalda lo antes mencionado.

Para el tejido óseo sano (+B) se presenta una situación similar a la anterior; se encuentra una diferencia en la deformación total máxima en la placa de bloqueo. Considerando el uso del soporte medial $(+\mathrm{A})$ se tiene un aumento del $4.8 \%$ (de $0.42 \mathrm{~mm}$ a $0.44 \mathrm{~mm}$ ) con un tejido óseo sano (+B), pudiendo tomar esta inconsistencia como trivial. A pesar de esto, se tiene que la mayoría de las fracturas de humero proximal están relacionadas con la osteoporosis [5, 6], por lo que es crítico el examinar estos resultados atendiendo esta condición. Al tomar como referencia el escenario propuesto en la Tabla $8(+\mathrm{A}+\mathrm{B}+\mathrm{C})$, se muestra que el tejido óseo osteoporótico (-B) experimenta un incremento de $3.6 \%$ (de $0.57 \mathrm{~mm}$ a $0.55 \mathrm{~mm}$ ) para la deformación total máxima y de $8 \%$ (de 11.4 MPa a 12.3 MPa) para el esfuerzo de von Mises. Asimismo, se expone un strain en hueso fracturado promedio de $6 \%$ catalogándose como un strain medio, el cuál es beneficioso según la teoría de strain interfragmentario.

Con respecto al material para la placa de bloqueo (C), se establece en la Tabla 8 que el acero inoxidable $(+C)$ es la mejor alternativa para su manufactura. En contraste, se aprecia una distinción en el esfuerzo de von Mises máximo para la placa de bloqueo. Al tomar como referencia el uso del soporte medial (+A) como del tejido óseo sano (+B), se muestra una intensificación mínima de la respuesta del sistema en un $4 \%$ (de 177.4 MPa a 184.4 MPa). A pesar de que estos resultados difieren de la propuesta planteada, se mostró en puntos anteriores que el utilizar titanio (-C) como material en el implante no permite cumplir con los valores de strain medio (2\% a $10 \%)$ que propone la teoría de strain interfragmentario, presentando valores máximos de hasta $17 \%$, lo que podría derivar en la no-unión y el fallo del sistema de fijación. El argumento anterior podría ofrecer un aspecto importante para la selección del Material de Placa (C) en el estudio. A partir de este punto de vista, el acero inoxidable $(+C)$ se elige como idóneo.

Con base a la información mostrada en el estudio, es posible argumentar que los resultados tomados en el modelo de placa de bloqueo podrían no ser tan importantes como los resultados obtenidos en el modelo del hueso fracturado debido a la diferencia notable en sus propiedades mecánicas (resistencia, módulo, etc.). Aunque el uso de un modelo isotrópico concluye en una estimación excesivamente conservadora de la carga necesaria para iniciar y propagar la fractura [54], es provechoso para el estudio de elementos biomecánicos, logrando conocer su significancia y permitir el establecer diferentes tendencias. Además, con la completa eliminación del hueso trabecular se logró simplificar el modelo y por consiguiente, reducir notablemente el tiempo de cálculo [46].

Con respecto a las posiciones de "levantar frontalmente un peso muerto" y en torsión, se tiene que los 
factores estudiados no mostraron relevancia significativa en el estudio, exceptuando dos escenarios: 1) el esfuerzo de von Mises máximo en placa de bloqueo para la primer posición y, 2) esfuerzo de von Mises máximo en hueso fracturado para torsión. En ambos casos, el factor B (Tipo de Carga) aparece como estadísticamente significativo, sugiriendo que el tejido osteoporótico (-B) mostraría un escenario favorecedor para minimizar la intensidad del esfuerzo según el caso. Se recomienda realizar en futuros trabajos un diseño de experimentos más robusto como el propuesto en la condición primeramente estudiada.

\section{CONCLUSIONES}

En este trabajo se ha analizado los elementos principales en el diseño de placas de bloqueo en una fractura de 2-partes del cuello quirúrgico del húmero utilizando MEF y análisis estadístico, buscando establecer un escenario óptimo. Estos hallazgos sugieren que, en general, el uso de un soporte medial en los sistemas de fijación con placas de bloqueo presenta el escenario más favorable para reducir el riesgo de fallo del sistema bajo las condiciones de carga descritas. De igual forma, si se considera que las placas de bloqueo son fabricadas de acero inoxidable, el resultado mejoraría considerablemente. Por otro lado, el tejido óseo (sano u osteoporótico) no muestra ser un elemento significativo sobre la variable de respuesta. Lo antes mencionado se toma al considerar los aspectos biomecánicos sobre los procesos de curación del hueso fracturado así como también de la misma fijación.

A pesar de que se obtuvo resultados relevantes en el área, es oportuno mencionar que los estudios biomecánicos son mayormente interdisciplinarios, por lo que requieren de habilidades en el área de la ingeniería, medicina y biología. Además, las metodologías utilizadas en este estudio siguen siendo muy útiles para definir una tendencia en los modelos biomecánicos con el fin de tomar mejores decisiones.

\section{AGRADECIMIENTOS}

Los autores agradecen a la Universidad Autónoma de Baja California por facilitar el acceso y uso de sus instalaciones y equipos para llevar a cabo esta investigación.

\section{BIBLIOGRAFÍA}

[1] PONCE, B. A., THOMPSON, K.J, RAGHAVA, P, et al., "The role of medial comminution and calcar restoration in varus collapse of proximal humeral fractures treated with locking plates", J. Bone Joint Surg. Am., v.95, n.16, 2013.

[2] AGUDELO, J., SCHÜRMANN, M, STAHEL, P, et al., "Analysis of Efficacy and Failure in Proximal Humerus Fractures Treated With Locking Plates", J Orthop Trauma, v. 21, n. 10, pp. 676-681, 2007.

[3] LESCHEID, J., ZDERO, R., SHAH, S., et al., "The biomechanics of locked plating for repairing proximal humerus fractures with or without medial cortical support.", J. Trauma, v. 69, n. 5, pp. 1235-42, 2010.

[4] NOBILE, F., CARTA, S., FORTINA, M., et al., "Displaced 3- and 4-part proximal humeral fractures: Evaluation and management with an intramedullary nail within $48 \mathrm{~h}$, in the emergency department", J. Acute Dis., v. 5, n. 2, pp. 154-159, 2016.

[5] PALVANEN, M., KANNUS, P., NIEMI, S., et al., "Update in the epidemiology of proximal humeral fractures", Clin Orthop Relat Res., v. 442, pp. 87-92, 2006.

[6] HESSMANN, M. H. STERNSTEIN, W., HANSEN, M., et al., "Locked plate fixation and intramedullary nailing for proximal humerus fractures: a biomechanical evaluation", J. Trauma-Injury Infect. Crit. Care, v. 58, n. 6, pp. 1194-1201, 2005.

[7] KWON, B., GOERTZEN, D., O'BRIEN, P., et al., "Biomechanical evaluation of proximal humeral fracture fixation supplemented with calcium phosphate cement", J Bone Jt. Surg Am., v. 84-A, n. 6, pp. 951-961, 2002.

[8] PONCE, B. A., THOMPSON, K.J, RAGHAVA, P., et al., "The role of medial comminution and calcar restoration in varus collapse of proximal humeral fractures treated with locking plates", J. Bone Joint Surg. Am., 2013.

[9] NEER, C. S. "Four-segment classification of proximal humeral fractures: Purpose and reliable use", $J$. Shoulder Elb. Surg., v. 11, n. 4, pp. 389-400, 2002.

[10] HAASTERS, F., SIEBENBÜRGER, G., HELFEN, T., et al., "Complications of locked plating for proximal humeral fractures-are we getting any better?", J. Shoulder Elb. Surg., v. 25, n. 10, pp. 295-303, Oct. 2016. 
[11] LANGE, M., BRANDT, D., MITTLMEIER, T., et al., "Proximal humeral fractures: non-operative treatment versus intramedullary nailing in 2-, 3- and 4-part fractures", Injury, v. 47, pp. S14-S19, 2016.

[12] ZHANG, L., ZHENG, J., WANG, W., et al., "The clinical benefit of medial support screws in locking plating of proximal humerus fractures: A prospective randomized study", Int. Orthop., v. 35, n. 11, pp. 16551661, 2011.

[13] SPROUL, R. C., IYENGAR, J. J., DEVCIC, Z., et al., "A systematic review of locking plate fixation of proximal humerus fractures", Injury, v. 42, n. 4, pp. 408-413, 2011.

[14] CHA, H., PARK, K. B., OH, S., et al., "Treatment of comminuted proximal humeral fractures using locking plate with strut allograft”, J. Shoulder Elb. Surg., v. 26, n. 5, pp. 781-785, 2017.

[15] CALORI, G. M., COLOMBO, M, BUCCI, M.S., et al., "Complications in proximal humeral fractures", Injury, v. 47, pp. 54-58, Oct. 2016.

[16] RÖDERER, G., GEBHARD, F., KRISCHAK, G., et al., "Biomechanical in vitro assessment of fixed angle plating using a new concept of locking for the treatment of osteoporotic proximal humerus fractures", Int. Orthop., v. 35, n. 4, pp. 535-541, 2011.

[17] SEIDE, K., TRIEBE, J, FASCHINGBAUER, M., et al., "Locked vs. unlocked plate osteosynthesis of the proximal humerus - A biomechanical study", Clin. Biomech., v. 22, n. 2, pp. 176-182, Feb. 2007.

[18] SIFFRI, P. C., PEINDL, R. D., COLEY, E. R., et al., "Biomechanical analysis of blade plate versus locking plate fixation for a proximal humerus fracture: comparison using cadaveric and synthetic humeri.", $J$. Orthop. Trauma, v. 20, n. 8, pp. 547-554, 2006.

[19] WEINSTEIN, D. M., BRATTON, D. R., CICCONE, W. J., et al., "Locking plates improve torsional resistance in the stabilization of three-part proximal humeral fractures", J. Shoulder Elb. Surg., v. 15, n. 2, pp. 239-243, 2006.

[20] LILL, H., HEPP, P., KORNER, J., et al., "Proximal humeral fractures: how stiff should an implant be? A comparative mechanical study with new implants in human specimens", Arch. Orthop. Trauma Surg., v. 123, n. 2-3, pp. 74-81, 2003.

[21] MOONOT, P., ASHWOOD, N., HAMLET, M., "Early results for treatment of three- and four-part fractures of the proximal humerus using the PHILOS plate system", J. Bone Joint Surg. Br., v. 89, n. 9, pp. 1206-9, 2007.

[22] LESCHEID, J., ZDERO, R., SHAH, S., et al., "The biomechanics of locked plating for repairing proximal humerus fractures with or without medial cortical support.", J. Trauma, v. 69, n. 5, pp. 1235-42, 2010.

[23] GARDNER, M. J., WEIL, Y., BARKER, J. U., et al., "The importance of medial support in locked plating of proximal humerus fractures", J. Orthop. Trauma, v. 21, n. 3, pp. 185-191, 2007.

[24] MILLER, D. L., GOSWAMI, T., "A review of locking compression plate biomechanics and their advantages as internal fixators in fracture healing", Clin. Biomech., v. 22, n. 10, pp. 1049-1062, 2007.

[25] GREIWE, R., ARCHDEACON, M., "Locking plate technology: current concepts", J Knee Surg., v. 20, no. 1, pp. 50-55, 2007.

[26] AHMAD, M., NANDA, R., BAJWA, A. S., et al., "Biomechanical testing of the locking compression plate: When does the distance between bone and implant significantly reduce construct stability?", Injury, v. 38, n. 3, pp. 358-364, 2007.

[27] ÖZÜTOK, A., MADENCI, E., "Static analysis of laminated composite beams based on higher-order shear deformation theory by using mixed-type finite element method", Int. J. Mech. Sci., v. 130, pp. 234-243, Sep. 2017.

[28] SAWACHA, Z., SCHREFLER, B., "Finite Element Modelling in Musculoskeletal Biomechanics", In: Modelling Methodology for Physiology and Medicine, 2nd ed., E. Cobelli and C. Carson, Eds. Newnes, 2014, pp. 527-544.

[29] GÍSLASON, M. K., STANSFIELD, B., NASH, D. H., "Finite element model creation and stability considerations of complex biological articulation: The human wrist joint", Med. Eng. Phys. J., v. 32, pp. 523$531,2010$.

[30] MASIH, C., NARELIYA, R., KUMAR, V., "Biomechanical Analysis of the RP Model of Human Humerus Bone and its Comparison with the Real Proximal Humeral Bone", In: Proceedings of All India Seminar on Biomedical Engineering 2012 (AISOBE 2012), v. 16, n. 1, pp. 87-97, 2013.

[31] KATTHAGEN, J. C., SCHWARZE, M., WARNHOFF, M., et al., "Influence of plate material and screw design on stiffness and ultimate load of locked plating in osteoporotic proximal humeral fractures", 
Injury, v. 47, n. 3, pp. 617-624, Mar. 2016.

[32] WEEKS, C. A., BEGUM, F., BEAUPRE, L. A., et al., "Locking plate fixation of proximal humeral fractures withimpaction of the fracture site to restore medial column support: A biomechanical study", $J$. Shoulder Elb. Surg., v. 22, n. 11, pp. 1552-1557, 2013.

[33] YAMAMOTO, N., HONGO, M., BERGLUND, L.J., et al., "Biomechanical analysis of a novel locking plate with smooth pegs versus a conventional locking plate with threaded screws for proximal humerus fractures", J. Shoulder Elb. Surg., v. 22, n. 4, pp. 445-450, 2013.

[34] SCHUMER R.A, MUCKLEY, K.L, MARKERT, R.J, et al., "Biomechanical comparison of a proximal humeral locking plate using two methods of head fixation”, J. Shoulder Elb. Surg., v. 19, n. 4, pp. 495-501, 2010 .

[35] FORURIA, A. M., CARRASCAL, M. T., REVILLA, C., et al., "Proximal humerus fracture rotational stability after fixation using a locking plate or a fixed-angle locked nail: The role of implant stiffness", Clin. Biomech., vol. 25, no. 4, pp. 307-311, 2010.

[36] MALDONADO, Z.M., SEEBECK, J., HELLER, M.O., et al., "Straining of the intact and fractured proximal humerus under physiological-like loading”, J. Biomech., v. 36, n. 12, pp. 1865-1873, Dec. 2003.

[37] DURALDE, X. A., LEDDY, L. R., "The results of ORIF of displaced unstable proximal humeral fractures using a locking plate”, J. Shoulder Elb. Surg., v. 19, n. 4, pp. 480-488, 2010.

[38] SABALIC, S., KODVANJ, J., PAVIC, A., "Comparative study of three models of extra-articular distal humerus fracture osteosynthesis using the finite element method on an osteoporotic computational model", Injury, v. 44, n. 3, pp. 56-61, Sep. 2013.

[39] YANG, P., ZHANG, Y., LIU, J., et al., "Biomechanical effect of medial cortical support and medial screw support on locking plate fixation in proximal humeral fractures with a medial gap: a finite element analysis”, Acta Orthop. Traumatol. Turc., v. 49, n. 2, pp. 203-209, 2015.

[40] HOLBERG, C., STEINHÄUSER, S., RUDZKI, I., "Surgically assisted rapid maxillary expansion: Midfacial and cranial stress distribution", Am. J. Orthod. Dentofac. Orthop., v. 132, n. 6, pp. 776-782, 2007.

[41] MANTOVANI, M., PELLEGRINI, A., GAROFALO, P., et al., "A 3D finite element model for geometrical and mechanical comparison of different supraspinatus repair techniques”, J. Shoulder Elb. Surg., v. 25, n. 4, pp. 557-563, Apr. 2016.

[42] OLDANI, C., DOMINGUEZ, A., "Titanium as a Biomaterial for Implants", In: Recent Advances in Arthroplasty, S. Fokte, Ed. InTech, pp. 149-162, 2012.

[43] OKEN, O. F., SOYDAN, Z., YILDIRIM, A O., et al., "Performance of modified anatomic plates is comparable to proximal femoral nail, dynamic hip screw and anatomic plates: finite element and biomechanical testing”, Injury, v. 42, n. 10, pp. 1077-83, 2011.

[44] CLAVERT, P., ZERAH, M., KRIER, J., et al., "Finite element analysis of the strain distribution in the humeral head tubercles during abduction: Comparison of young and osteoporotic bone", Surg. Radiol. Anat., v. 28 , n. 6, pp. 581-587, 2006.

[45] SERAL, B., GARCÍA, J. M., CEGOÑINO, J., et al., "Finite element study of intramedullary osteosynthesis in the treatment of trochanteric fractures of the hip: Gamma and PFN", Injury, v. 35, n. 2, pp. 130-135, 2004.

[46] BOGATAJ, M., KOSEL, F., NORRIS, R., KRKOVIC, M., and BROJAN, M., "Biomechanical study of different plate configurations for distal humerus osteosynthesis", Med. Biol. Eng. Comput., v. 53, n. 5, pp. 381-392, May 2015.

[47] DAHAN, G., TRABELSI, N., SAFRAN, O., et al., "Verified and validated finite element analyses of humeri”, J. Biomech., pp. 1-9, 2016.

[48] ZHANG, Y.K., WEI, H.W., LIN, K.P., et al., "Biomechanical effect of the configuration of screw hole style on locking plate fixation in proximal humerus fracture with a simulated gap: A finite element analysis", Injury, v.47, n.6, pp. 1191-1195, 2016.

[49] INZANA, J. A., VARGA, P., WINDOLF, M., "Implicit modeling of screw threads for efficient finite element analysis of complex bone-implant systems", J. Biomech., v. 49, n. 9, pp. 1836-1844, Jun. 2016.

[50] JIJU, A., Design of Experiments for Engineers and Scientists, 2nd ed. 2014.

[51] DAR, F. H., MEAKIN, J. R., ASPDEN, R. M., "Statistical methods in finite element analysis", J. Biomech., v. 35, n. 9, pp. 1155-1161, 2002.

[52] KALA, M., SHAIKH, M. V., NIVSARKAR, M., "Development and optimization of psychological 
stress model in mice using 2 level full factorial design”, J. Pharmacol. Toxicol. Methods, v. 82, pp. 54-61, 2016.

[53] GUTIÉRREZ PULIDO, H., ROMÁN DE LA VARA, S., Análisis y diseño de experimentos, 2nd ed. Distrito Federal, México: McGraw-Hill Interamericana, 2008.

[54] URAL, A.VASHISHTH, D., "Cohesive finite element modeling of age-related toughness loss in human cortical bone”, J. Biomech., v. 39, n. 16, pp. 2974-2982, 2006. 